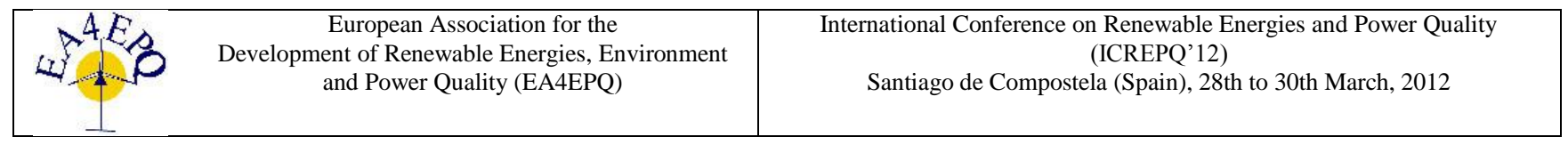

\title{
Fuzzy Coordinated Control of TCSCs to Improve Power System Stability
}

\author{
A. Ghafouri ${ }^{1}$, G. B. Gharehpetian ${ }^{2}$, J. Milimonfared ${ }^{2}$ \\ ${ }^{1}$ Department of Electrical Engineering - Sari Branch - Islamic Azad University- Sari - Iran \\ Phone number: +98 9113554835, e-mail:ghafouri@iausari.ac.ir \\ ${ }^{2}$ Amirkabir University of Technology \\ Electrical Engineering Department,Tehran, Iran \\ Phone number: +98 64543341, e-mail: grptian@aut.ac.ir, monfared@aut.ac.ir
}

\begin{abstract}
This paper presents the application of a coordinated fuzzy logic controller for the Thyristor Controlled Series Capacitors (TCSC) to improve the stability of the power system. The nonlinear fuzzy logic controller is used to overcome the problems generated by modeling and different uncertainties existing in power systems during design stage of controllers to damp electromechanical oscillations. To facilitate the design process, neural network is used to model the system input-output behavior. Proposed method is implemented on the 9 Bus IEEE power system and simulation results are presented to confirm the performance of the proposed controller. It is shown that this controller can increase the damping of the system.
\end{abstract}

\section{Key words}

Power System Stability, Fuzzy Logic Controller, TCSC, Neural Network, Oscillation damping.

\section{Introduction}

Power systems are becoming rapidly more complex because of deregulation, interconnection of regional systems and fast growing demand. This situation obliges new considerations to maintain stability and reliability of the system. Recently, FACTS devices are introduced to face these problems and improve the performance of power systems. They can improve controllability of active and reactive powers. Using proper controllers, FACTS devices are also able to ameliorate the dynamic behavior of the system, in the vein of transient, small signal and voltage stability.

Different methods have been used for stability analysis in power systems. Time domain simulation and direct method are two important methods that have some benefits and shortcomings. In the time domain simulation, power system elements are modeled in proper software and stability is checked for each contingency. As simulation is based on the model of the system, the more exact the model, the more actual the results if the complexity of the system does not provoke numerical problems. Simulation time is also an important issue in this type of analysis and in many cases limits the application of the online study. These problems become more important when a larger system is being studied. In direct method there is no need to solve the dynamic equations of the system, so it is faster than time domain simulation. This method is mainly based on Lyapunov stability analysis theory and needs to form the system energy function as a Lyapunov function [1]. This method is used for transient stability analysis and controller design. If energy function is derived, power system stability can be checked by monitoring this function and its rate of descent. Model based characteristics and the need to several measured parameters are the main shortcoming of this method [2]. Linear time invariant methods are applicable for small signal stability analysis and controller design, especially state feedback and pole placement. Recently, some researchers have performed these method using adaptive or robust techniques to decrease the model based characteristic and use some local measurements to obtain stability criteria and design a control strategy [3, 4].

Flexible AC Transmission System (FACTS) devices are used to control power flow along transmission lines and improve power system stability [5]. Thyristor Controlled Series Capacitor (TCSC) is one of the series FACTS devices that are usually used to control power flow in transmission lines. It is also used to improve power system stability by adjusting the reactance of transmission line. This function of TCSC needs some more supplementary input signals [6]. Several controllers have been used to perform this control strategy such as conventional PI controller [6], rule based controller [7] and energy function based controller [8]. In a power system, coordinated control of different devices is one of the most important considerations. This problem becomes more important if the number of FACTS devices increases. In [10] and [11], some coordination methods are presented based on linear model of the system.

Uncertainty exists in almost every physical system. It can be the result of different phenomena according to system 
nature, lake of information or measurements. Power systems are large scale systems with high nonlinearity, so there is a considerable uncertainty in every part of them. Fuzzy logic performs as a powerful tool to confront these uncertainties [9].

This paper presents a coordinated fuzzy logic based control strategy for TCSCs to improve power system stability. The fuzzy logic approach provides a model-free method for TCSC control and can be effective over a wide range of power system changes. Fuzzy logic approach allows the designer to incorporate experimental knowledge in adjustment of controller parameters. Selection of proper control signals for Fuzzy Logic Controller (FLC) is very important because controller design, system response and sensitivity depend directly on it. In this work, the frequency of generators is chosen as inputs. To improve the controller performance, a neural network model of power system is trained based on data derived from the system in different conditions.

In part II, TCSC model is presented for stability analysis and its effect on stability is expressed. In part III some fuzzy logic principles and control strategies are introduced. This strategy is based on Mamdani's FLC. The advantage of this controller is that it doesn't need detailed information about the system. Also, structure of the neural network and its implementation is described in this section. In part IV, the proposed method is implemented on a sample network and simulation results are discussed.

\section{TCSC Modeling}

TCSC can be represented by a controlled series reactance as shown in Fig.1 [5].

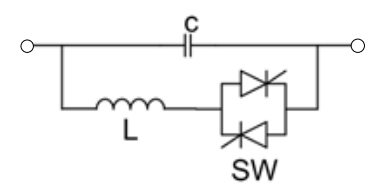

Fig. 1 Simple structure of TCSC

As it is shown in Fig.1, TCSC is a variable inductance in parallel with a fixed capacitor. So, the impedance of TCSC can be written, as follows:

$$
X_{T C S C}(\alpha)=\frac{X_{C} X_{L}(\alpha)}{X_{L}(\alpha)-X_{C}}
$$

where

$$
X_{L}(\alpha)=X_{L} \frac{\pi}{\pi-2 \alpha-\sin \alpha}
$$

and $\alpha$ is the delay angle measured from the crest of the capacitor voltage. The variation of $\mathrm{X}_{\mathrm{TCSC}}$ according to $\alpha$ is shown in Fig. 2.

In the vein of stability, TCSC is a variable impedance, so it can be used to increase the controllability of the system. TCSC can change the active power curve in the single machine infinite bus system, so the active power transfer can be controlled. Fig. 3 shows the effect of TCSC on the transferred power of generator for single machine infinite bus (SMIB) system graphically.

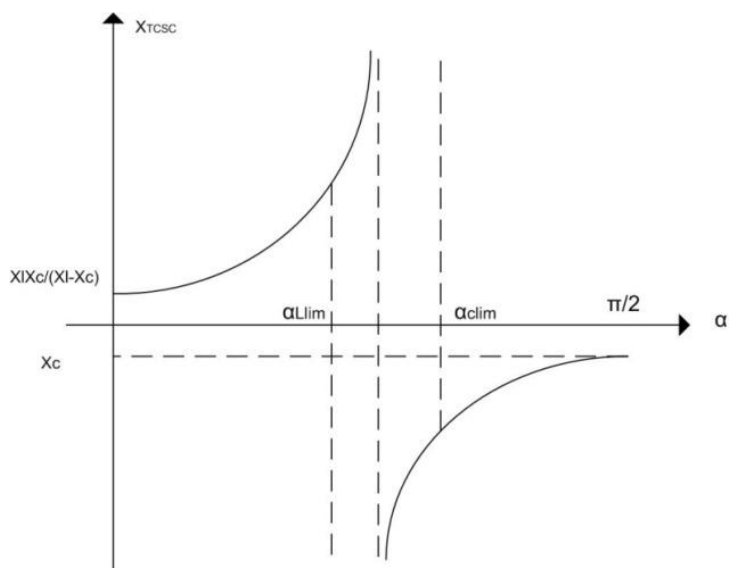

Fig. 2. TCSC model as variable reactance

The maximum transferred power of generator can be increased when TCSC is in the capacitive mode and decreased when it is in the inductive mode. Proper controller can adjust TCSC to improve stability margin of the system.

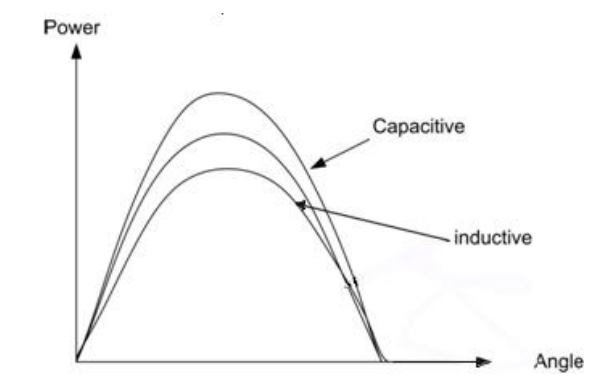

Fig. 3. The effect of TCSC on electrical power curves

\section{Control Strategy}

In this section a control strategy of TCSC is developed for the damping of electromechanical oscillations. First, the neural network structure and its training are expressed. Then fuzzy controller is designed and its parameters adjustment is done. Finally, a controller is proposed based on the fuzzy controller structure.

\section{A. Neural Network Model of Power System}

In this paper, a three layer feed-forward neural network is used. Input signals are TCSCs reactance and output signals are frequencies of generators. Fig. 4 shows the structure of proposed neural network [15].

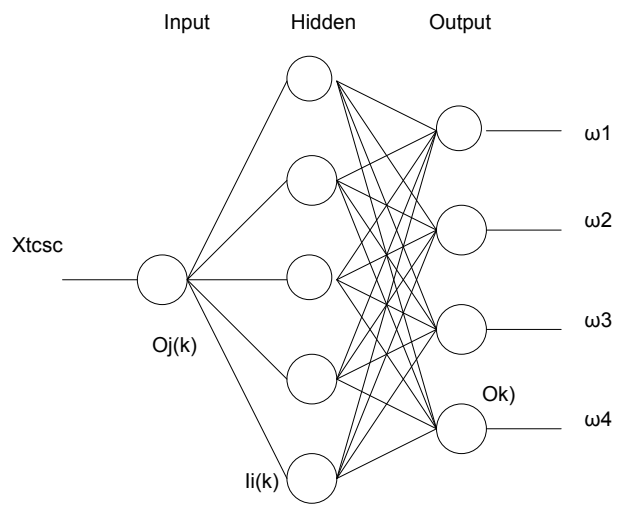

Fig. 4 Proposed Neural Network to model the effect of TCSCs on generators frequency 
In hidden layer, the sigmoid function is utilized for the input-output characteristics of the nodes. For each node $i$ in the hidden and output layer, the output $O_{i}(k)$ is given as follows [15]:

$$
O_{i}(k)=\frac{1}{1+e^{-I_{i}(k)}}
$$

$I_{i}(k)$ is the input signal to node $i$ at the $k$ th sampling and is given by the weighed sum of the input nodes as follows:

$$
I_{i}(k)=\sum_{j} w_{i j}(k) O_{j}(k)
$$

Where $w_{i j}$ is the connection weight from node $j$ to node $i$ and $O_{j}(k)$ is the output from node $j$.

In the training process, a set of input-output patterns for neural network is required. During the training, the connection weights are modified recursively until the best fit is achieved based on the minimum value of square sum of errors as

$$
S S E=\sum_{k=1}^{N}(t(k)-O(k))^{2}
$$

Where $N$ is the total number of training patterns, $t(k)$ is the $k t h$ target output and $O(k)$ is the computed value.

\section{B. Fuzzy Logic Controller}

The proposed fuzzy controller is based on mamdani's controller, which uses "if-then" rules for inference engine [12]. A fuzzy variable is considered for each controller input and these inputs are fuzzified by proper membership functions. Crisp output is calculated by Center of Area (COA) method. Proper membership functions are defined for output variables, too. Linguistic variable definition is based on effectiveness of the variable on stability criteria. In this work, input variables are frequency of generators that are mostly affected by TCSCs. Fig. 5 shows the membership functions assigned to each input variable.

The proper range for each term and the number of membership functions can be defined based on designer experiments and the system configuration. Actually, power system is confronted with several disturbances and frequency oscillation almost everywhere. Zero membership function determination is very important because TCSC should not respond to usual oscillations, which are not derived from disturbances related to instability problem.

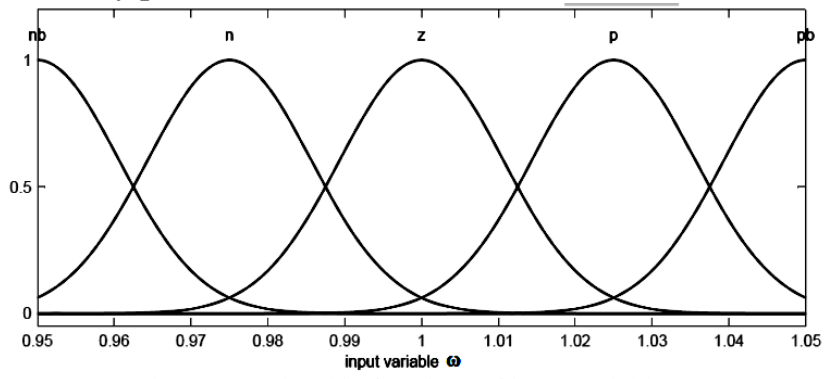

Fig. 5. Membership functions of input variables

The method to adjust input membership functions is based on the fact that the worst condition in transient stability is when the fault occurs at the generator bus or at the sending end of transmission line. So, the range of frequency deviation can be derived by applying the fault at the terminal buses of the network.

Fig.6 shows the output membership functions, which are used to build controller crisp output from the fuzzy outputs of inference engine. The deffuzzification method used for this purpose is COA [12]. In this paper, the fuzzy controllers have five outputs; each for one TCSC.

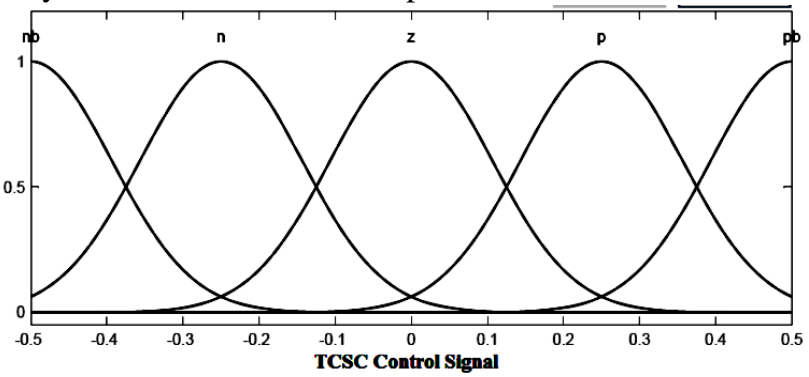

Fig. 6. Output membership functions

The fuzzy control strategy is realized in the inference engine that is a rule base containing all possible combinations of inputs and proper outputs for each of them [12]. Table I shows the rule base of the proposed FLC1 with $\omega_{1}$ as input signal and reactance of TCSCs as outputs. Similar fuzzy controllers are developed for other generator and their rule bases are designed based on the neural networks identified in the previous section.

Final control signal for TCSCs can be coordinated as weighted sum of control signals from each controller.

Table I. Rule base of FLC1

Outputs for $\omega_{1}$

\begin{tabular}{|l|l|l|l|l|l|}
\hline Xtcsc & nb & n & z & p & pb \\
\hline 1 & nb & n & z & p & pb \\
\hline 2 & nb & nb & z & pb & pb \\
\hline 3 & n & z & z & p & p \\
\hline 4 & p & z & z & z & n \\
\hline 5 & p & Z & z & z & n \\
\hline
\end{tabular}

Based on input variables $\left(\omega_{1}, \omega_{2}, \omega_{3}, \omega_{4}\right)$, outputs (the TCSCs reactance) are determined. Its capacitive and inductive mode is determined by the sign of output variable. The logic of rule definition can be derived as follows. For example:

$\mathrm{R} 1$ : If $\omega_{2}$ is positive big, then the TCSC1 reactance should be positive big (capacitive),TCSC2 reactance should be negative (inductive), TCSC 3 reactance should be negative (inductive), TCSC4 reactance should be positive (capacitive), TCSC5 reactance should be negative (inductive).

$\mathrm{R} 2$ : If $\omega_{2}$ is negative big, then the TCSC1 reactance should be negative big (inductive), TCSC2 reactance should be positive (capacitive), TCSC 3 reactance should be negative (inductive), TCSC4 reactance should be negative (inductive), TCSC5 reactance should be negative (inductive).

The logic is that when frequency is high and it is rising fast, the system is in a critical condition because the input mechanical power of generator is more than output electrical power. Therefore, the TCSCs should change 
the line reactance and so the power flows to increase the stability.

\section{SIMULATION RESULTS}

The proposed controller is implemented by Fuzzy Logic Toolbox of Matlab/Simulink and it is linked to PSCAD, in which the 9-bus IEEE system is implemented. Results, obtained from the system, are described in the following paragraphs.

\section{Simulation Results for IEEE 9-BusPower System}

Single line diagram of the IEEE 4-machine, 9-bus power system [13] is shown in Fig. 7. Five TCSCs are installed on lines 6-7, 7-8, 5-7, 5-6 and 8-9 of the system.

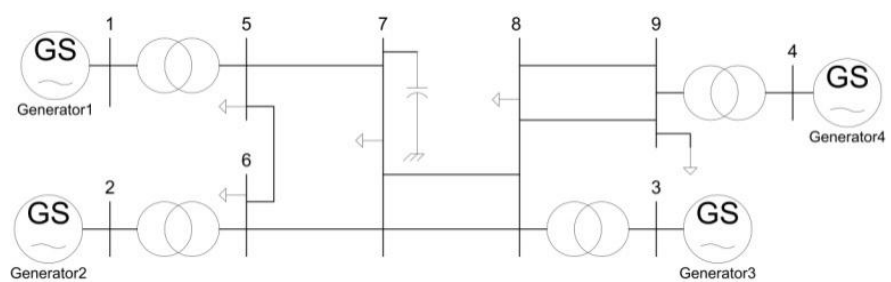

Fig. 7. IEEE 9-Bus single line diagram

A three phase fault on bus 7 is cleared after $100 \mathrm{~ms}$ with opening of line 7-8. Fig. 8 shows machine angles with respect to generator 4 without using any controller for TCSCs.

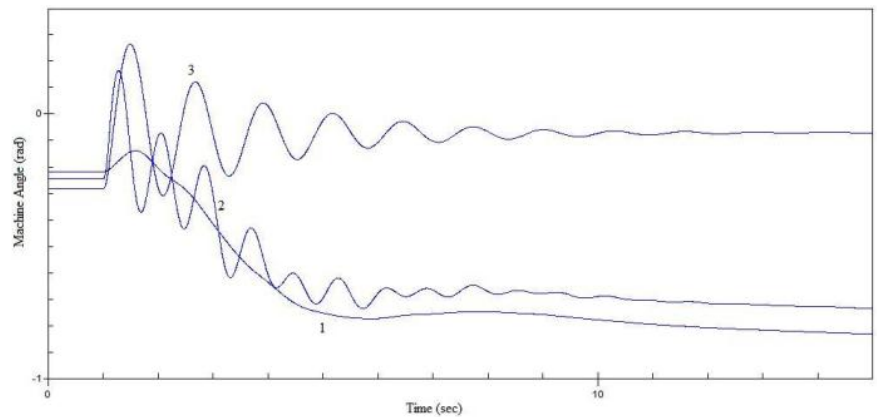

Fig. 8. Swing curves of IEEE 9-bus system

As it is shown, system goes to a new equilibrium point because fault clearing is the result of line 7-8 disconnection and the system configuration is changed. Fig. 9 shows the frequency of generators after occurrence of this fault.

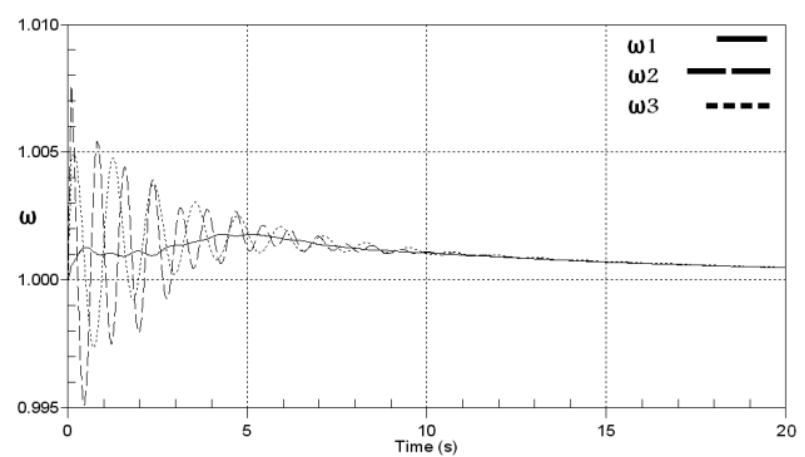

Fig. 9. Frequency of generators after fault occurrence at bus \#7 and opening of line 7-8
Power system is highly nonlinear so it is difficult to model it with classical methods. Neural networks can provide a proper model for these systems. To train neural networks, reactance of each TCSC is changed as input signal and the frequencies of generators are derived as outputs. So, five neural networks are trained as shown in Fig. 10. These NNs show the effect of TCSCs on the frequency of generators. Fuzzy logic controller can be designed based on these NNs for different conditions of the system. For example, when $\omega_{1}$ is increasing rapidly from a specific point, the controller should:

$$
\begin{aligned}
& \text {-decrease } \mathrm{X}_{\mathrm{TCSC} 1} \text { - decrease } \mathrm{X}_{\mathrm{TCSC} 2} \text { - decrease } \mathrm{X}_{\mathrm{TCSC} 3} \\
& \text { - increase } \mathrm{X}_{\mathrm{TCSC} 4} \text { - increase } \mathrm{X}_{\mathrm{TCSC} 5} \\
& \stackrel{\text { Fig. 10. NNs trained to show the effect of }}{\longrightarrow} \\
& \text { each TCSC on generators frequency }
\end{aligned}
$$

So we will have four controllers for each generator. The final control signal can be calculated as follows:

$\mathrm{X}_{\mathrm{TCSC} 1}=\mathrm{a}_{1} \mathrm{X}_{\mathrm{TCSC} 1 \omega 1}+\mathrm{a}_{2} \mathrm{X}_{\mathrm{TCSC} 1 \omega 2}+\mathrm{a}_{3} \mathrm{X}_{\mathrm{TCSC} 1 \omega 3}+\mathrm{a}_{4} \mathrm{X}_{\mathrm{TCSC} 1 \omega 4}$

where

$\mathrm{X}_{\mathrm{TCSCi} \omega \mathrm{j}}$ is the reactance of TCSCi derived from controller of generator $\mathrm{j}$.

$a_{1}, a_{2}, \ldots, a_{5}$ are constant coefficients or weights of control signals and are calculated in (7).

$$
a_{i}=\frac{\Delta \omega_{\text {imax }}}{\sum_{k=1}^{4} \Delta \omega_{k \max }}
$$

$\Delta \omega_{\text {imax }}$ is the maximum frequency deviation of generator $i$ after three phase short circuit fault on different buses of system. Block diagram of the system is shown in Fig. 11.

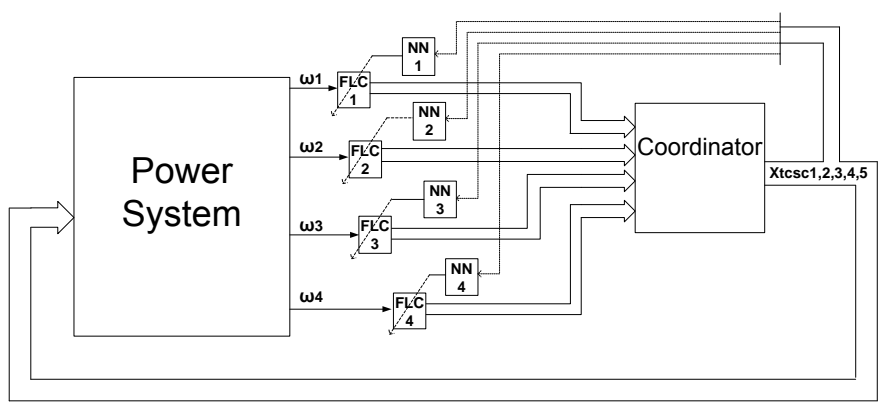

Fig. 11. Structure of control system

To study the effect of controller on stability of the system, three phase fault is placed on different buses of the system. As it is shown in figures 12-20 proposed controller could considerably improve the stability of generators and low frequency oscillations are rapidly damped. It should be mentioned that dashed lines are related to uncontrolled frequency curves. 


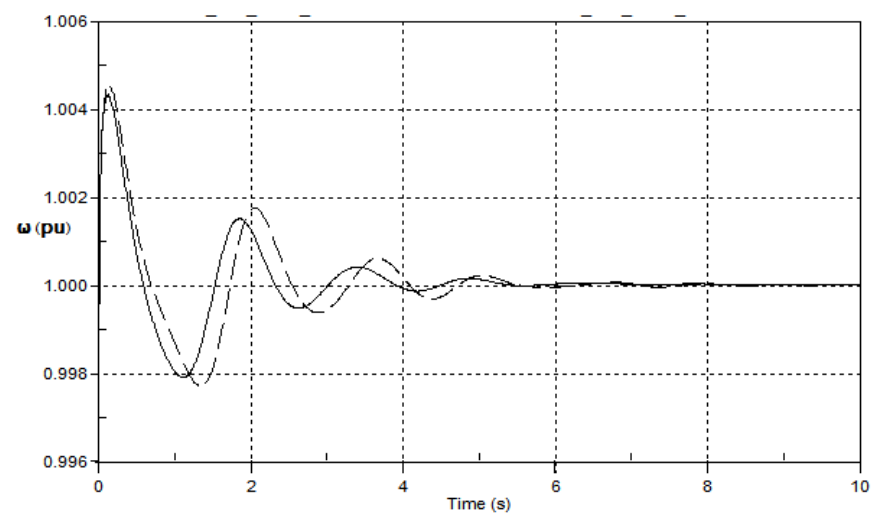

Fig. 12. Frequency of generator 2 after fault at bus \#6 and opening of line 5-6

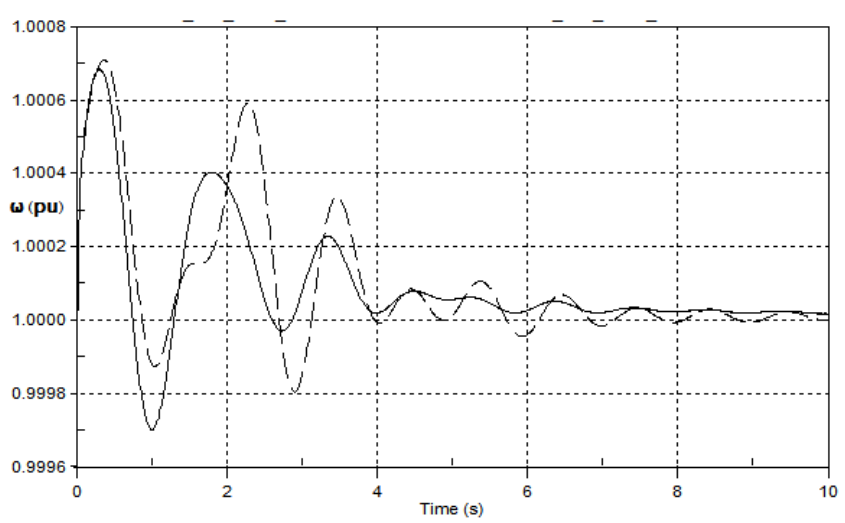

Fig. 13. Frequency of generator 3 after fault at bus \#6 and opening of line 5-6

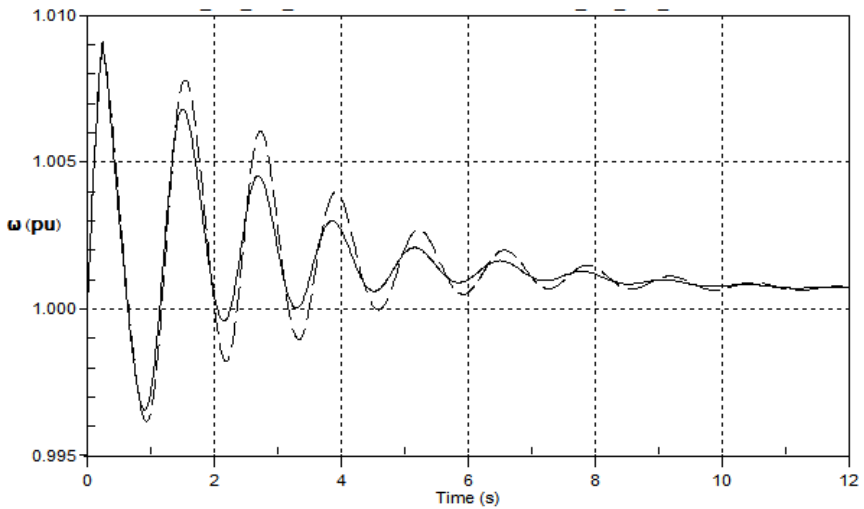

Fig. 14. Frequency of generator 3 after fault at bus \#7 and opening of line 7-8

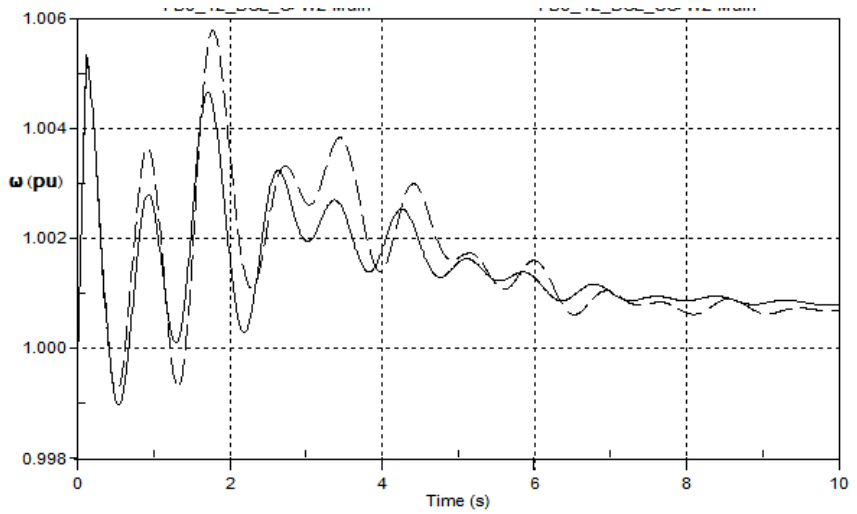

Fig. 15. Frequency of generator 2 after fault at bus \#8 and opening of line 7-8

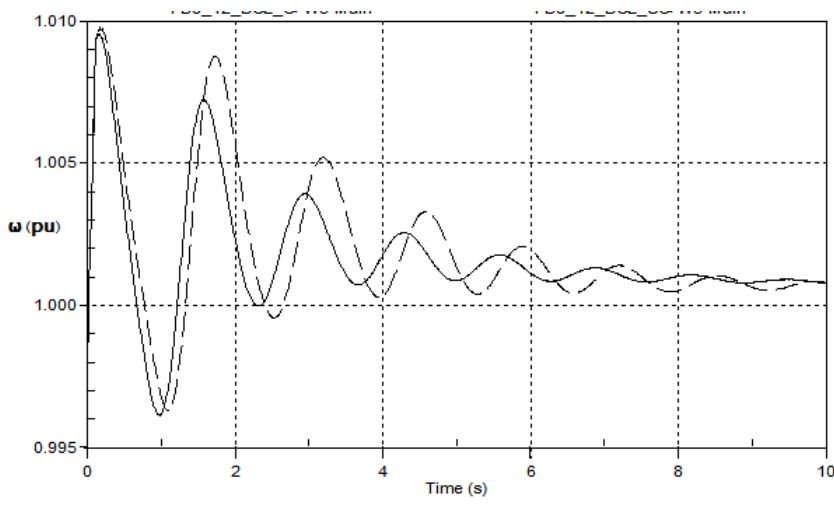

Fig. 16. Frequency of generator 3 after fault at bus \#8 and opening of line 7-8

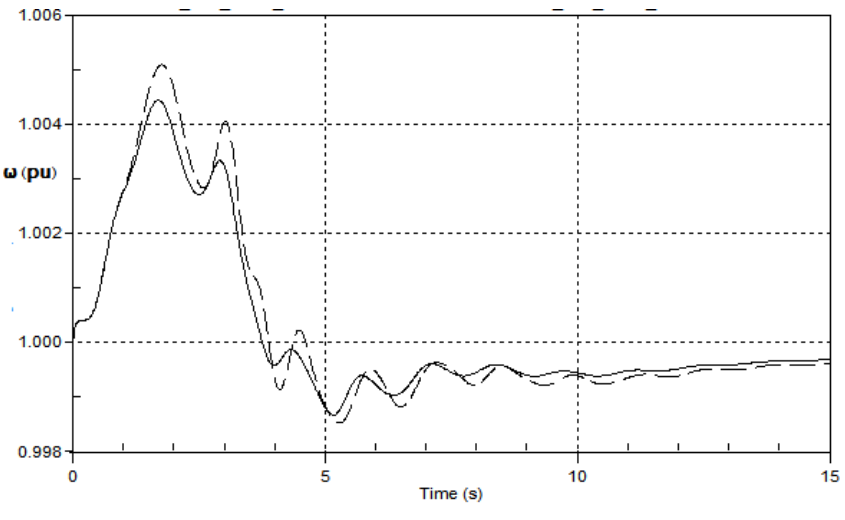

Fig. 17. Frequency of generator 2 after fault at bus \#9 and opening of line 8-9

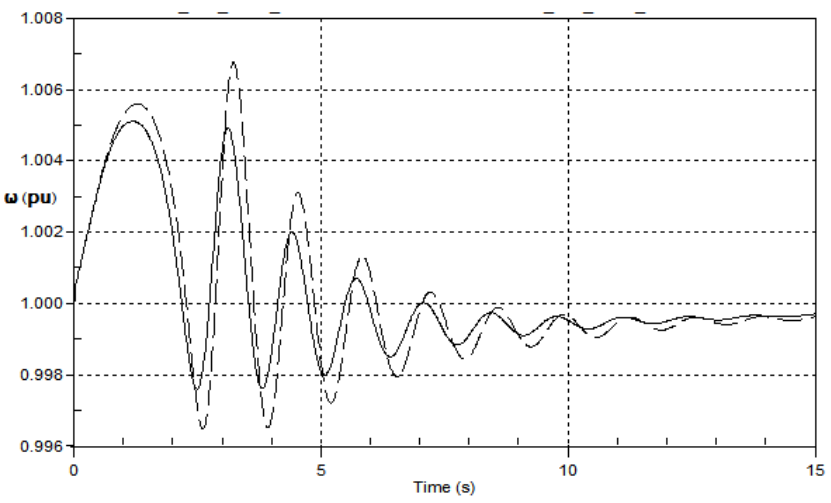

Fig. 18. Frequency of generator 3 after fault at bus \#9 and opening of line 8-9

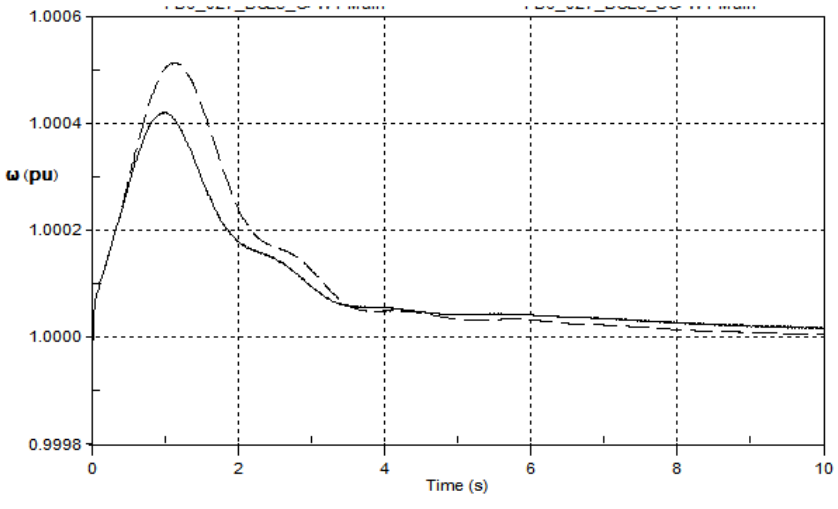

Fig. 19. Frequency of generator 1 after fault at bus \#6 and opening of line 5-6

https://doi.org/10.24084/repqj10.525

917

RE\&PQJ, Vol.1, No.10, April 2012 


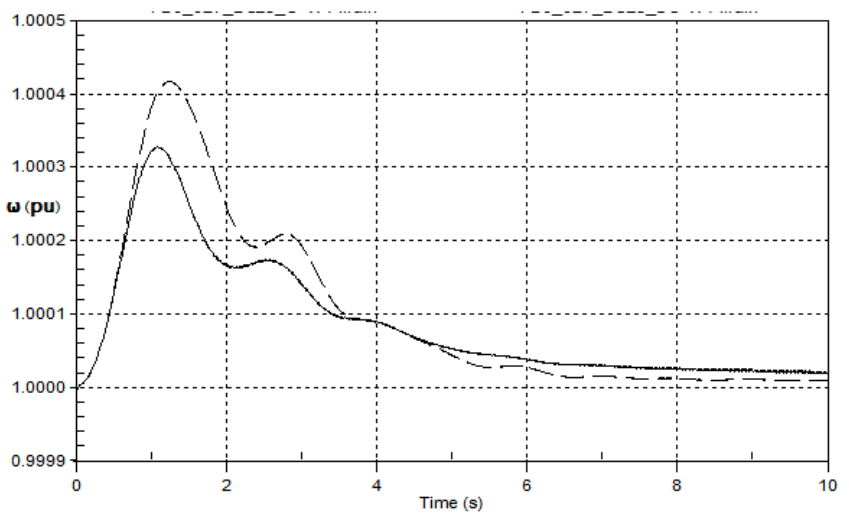

Fig. 20. Frequency of generator 4 after fault at bus \#6 and opening of line 5-6

To show that the controller improves the stability of the whole system, a criterion is defined as the difference of the mechanical input power to the system and electrical output power that is the kinetic energy of the whole machines. This energy is calculated respect to the steady state condition, as follows [14]:

$$
K E=\frac{1}{2} \sum_{i=1}^{n} M_{i}\left(\omega_{i}-\omega_{0}\right)^{2}
$$

where $M_{i}$ is the inertia constant of the generator $i$ and $\omega_{0}$ is:

$$
\begin{aligned}
& \omega_{0}=\dot{\delta}_{0} \\
& \delta_{0}=\frac{1}{M_{T}} \sum_{i=1}^{n} M_{i} \delta_{i} \quad, \quad M_{T}=\sum_{i=1}^{n} M_{i}
\end{aligned}
$$

In a power system, the difference between mechanical input power and electrical output power leads to frequency deviation and power oscillations. In the equilibrium point, these two powers are equal for the whole system. When a fault occurs, these powers will not stay in an equivalent condition and the kinetic energy will change. To evaluate the performance of the controller, kinetic energy of rotors $(K E)$ is shown in Fig. 21 after a fault on bus 7. According to this figure, with the proposed controller, kinetic energy of the total system decreases rapidly with less oscillation. This proves the ability of the proposed controller in power system stability improvement. Same results can be achieved for the fault on other buses in the network.

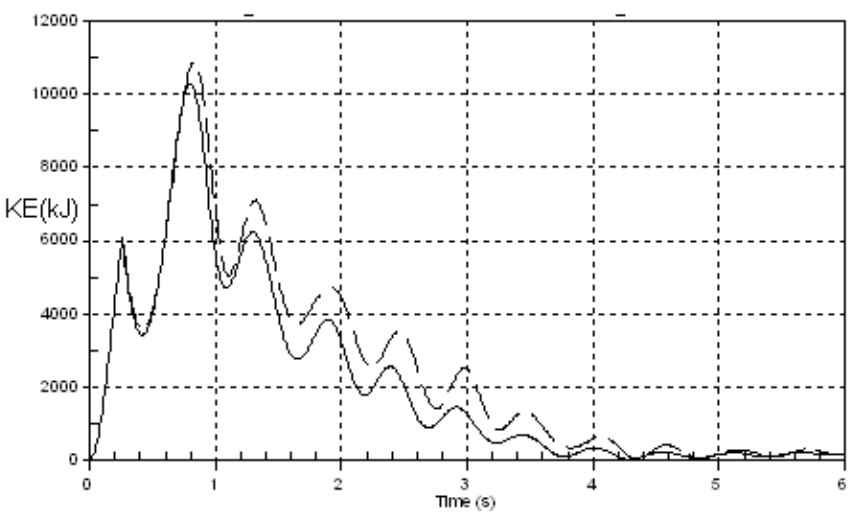

Fig. 21. KE for fault on bus 7

\section{Conclusions}

In this paper, a coordinated fuzzy logic controller has been proposed to control TCSCs to improve power system stability and damping. Fuzzy logic controller input parameters have been chosen carefully to provide considerable damping for the power system. The range of controller inputs and their rule base has been determined based on NNs trained by simulation data. Proposed control strategy has been applied to the IEEE 9-Bus power system with five TCSCs. Simulation results indicate that this controller can improve the stability of the system and provide considerable damping of power oscillation.

\section{References}

P.Kundur, 'Power system stability and control',McGraw-Hill, 1994 Pravin Varaiya, Felix F., and Rong-Liang Chen, "Direct Methods for Transient Stability Analysis of Power Systems: Recent Results," Proceedings of the IEEE, Vol..73, No..12, pp. 1703-1715, December 1985.

T. Athay, R. Podmore, and S. Virmani, "A Robust Control Strategy for Shunt and Series Reactive Compensators to Damp Electromechanical Oscillations," IEEE Trans. Power Deliveryt., Vol. 16, No. 4, pp. 812-817, October. 2001.

H. F. Wang, "Selection of Robust Installing Locations and Feedback Signals of FACTS-based Stabilizers in Multi-machine Power Systems," IEEE Trans. Power System, vol. 14, No. 2, pp. 569574, Nov. 1999.

Higorani, N. G., Gyugyi, L., “Understanding FACTS Devices,” IEEE Press 2000

Z. Huaang, Y. X. Ni, C. M. Shen, F. F. Wu, S. Chen, and B. Zhang, "Application of Unified Power Flow Controller in Interconnected Power Systems- Modeling, Interface, Control Strategy, and Case Study," IEEE Trans. Power System, vol. 15, pp. 811-816, May 2000

Chuen-Tsai Sun, "Rule-Base Structure Identification in an AdaptiveNetwork-Based Fuzzy Inference System," IEEE Trans. Fuzzy Systems, Vol. 2, No. 1, pp. 64-73, February 1994.

M.H. Haque, "Use of Energy Function to Evaluate the Additional Damping Provided by a STATCOM," Electric Power Systems Research , vol. 72, pp. 195-202, 2004

Langari Reza, "Fuzzy Control Synthesis and Analysis," Wiley Publications, 1995

Li-Jun Cai, Estivan Erich, "Simultaneous Coordinated Tuning of PSS and FACTS Damping Controllers in Large Power Systems", IEEE Trans. Power System, vol. 20, No. 1, pp.2949-300, Feb. 2005.

Gabriela Hug-Glanzman,Goran Anderson "Coordinated control of facts devices in power systems for security enhancement", iRep Symposium, Bulk power system dynamics and control, Charleston, USA, Aug. 2007.

Zimermann H. J (Hans-Jurgen), Fuzzy Set Theory and it's Applications, $3^{\text {rd }}$ Edition, 1996.

Available online at: http://psdyn.ece.wisc.edu/IEEE_benchmarks/

T. Athey, R. Podmore, S. Virmani,'A practical method for direct analysis of transient stability', IEEE Trans. Power Apparatus, vol. Pas-98, No. 2, pp.573-586, March/April 1979.

Syafaruddin, E. Karatepe, T. Hiyama,'Artificial neural network-polar coordinated fuzzy controller based maximum power point tracking control under partially shaded conditions', IET Renew. Power Gener., 2009, Vol. 3, Iss. 2, pp. 239-253 